\title{
The spectrum of prevention: developing a comprehensive approach to injury prevention
}

\author{
Larry Cohen, Susan Swift
}

\begin{abstract}
Objective-The purpose of this paper is to describe the "spectrum of prevention", a framework for developing multifaceted approaches to injury prevention. The value of the tool is that it can help practitioners develop and structure comprehensive initiatives.

Methods-The spectrum is comprised of six inter-related action levels: (1) strengthening individual knowledge and skills, (2) promoting community education, (3) educating providers, (4) fostering coalitions and networks, (5) changing organizational practices, and (6) influencing policy and legislation. Activities at each of these levels have the potential to support each other and promote overall community health and safety.

Conclusions-The spectrum of prevention is a tool which can help practitioners and policy leaders move beyond a primarily educational approach to achieve broad community goals through injury prevention strategies that include policy development. This framework has been endorsed and applied in a variety of disciplines, however it has not been formally evaluated, a process that could clarify the scope of its effectiveness.

(Injury Prevention 1999;5:203-207)
\end{abstract}

Keywords: spectrum of prevention; comprehensive approach; coalition building

The purpose of this paper is to describe the "spectrum of prevention", a tool for developing a multifaceted approach to injury prevention, and to encourage practitioners to implement comprehensive initiatives. The tool is comprised of six levels of increasing scope (see fig 1) beginning with a focus on the individual and family, on community norms, institutional practices, and finally laws. The spectrum seeks to aid practitioners to reduce injuries and their severity by identifying the need for a systems approach and encouraging an overall strategy which can result in "a whole that is greater than the sum of its parts".

As Rivara and Mueller point out, "until recently there has been little interest in developing conceptual models for injury research. The lack of systematic approaches has resulted in little continuity between studies or in progress toward a better understanding of the "best" solutions to the injury problem. The present haphazard approach must be replaced by more rational and scientific analysis".

The spectrum of prevention was developed in 1983 by Larry Cohen for a prevention training video, Beyond Brochures, and based upon the clinical work of Dr Marshall Swift from Hahnemann College in developmental disability prevention. It emerged from the conviction that preventive practice was too frequently trivialized and misunderstood as simply an educational practice. This spectrum tool was derived from practice and developed out of the conviction that complex problems require comprehensive solutions. The use of the tool was refined in the practice of a local health department - the prevention program directed by Larry Cohen in Contra Costa County, California, United States-where it was implemented in injury prevention for children and adolescents. $^{2}$

Perhaps the most important tool in the injury prevention field is the Haddon matrix. ${ }^{3}$ Haddon contributed immensely by distinguishing between prevention efforts that take place before the injury occurs from those implemented after the injury, that serve only to reduce the severity of trauma. The spectrum supplements the Haddon matrix as it helps practitioners to specify the array of activities necessary for an effective prevention campaign. By using the two tools together, practitioners can devise a multifactored intervention that simultaneously addresses the temporal issues highlighted by the Haddon matrix (that is multiple strategies for before, during, and after an injury event). The spectrum emphasizes the importance of influencing policy and legisla-

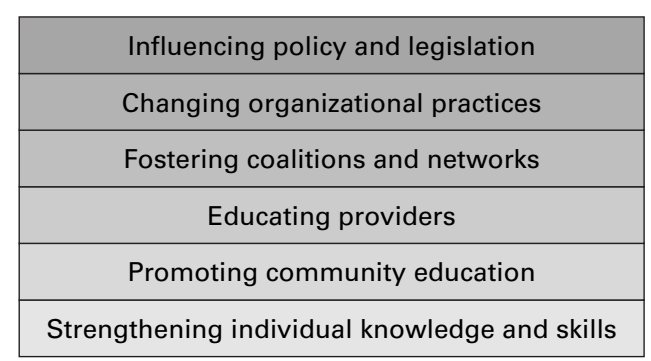

Figure 1 The spectrum of prevention.

Prevention Institute, Berkeley, CA 94707 USA

S Swift 
tion, an area which Haddon's approach does not specifically address.

\section{Prevention is more than education and goes beyond the individual}

Attempts to prevent childhood injuries with only medical interventions or educational materials can be expensive and insufficient. As public health expert George Albee stated, "...no mass disorder afflicting mankind is ever brought under control or eliminated by attempts at treating the individual or by attempts at producing large numbers of individual practitioners". "Many begin their plans to prevent injuries to children and adolescents by considering only education aimed at changing individual behaviors. Such an approach often fails to achieve maximum success. Videos, brochures, and newsletters flood physician waiting rooms. On-line computer services, radio, television, and the mail deliver information on health problems. Such materials, for instance, encourage individuals to quit drinking, to exercise, and to use car seats and seat belts. These personal health messages reinforce the commonly held misconception that individual behaviors are solely responsible for health outcomes, and therefore that individual health education is an adequate solution. Effective prevention is not that simple. The spectrum shifts attention from individually focused health education to a systems approach.

\section{Value of a comprehensive approach in injury prevention}

A new discipline of prevention is emerging. It embraces comprehensive strategy development coupled with interdisciplinary collaboration. The following example illustrates the way in which multiple activities, each corresponding with a level of the spectrum, can be implemented simultaneously to comprise a comprehensive drowning prevention approach. Early drowning prevention relied upon brochures, presentations, and training in cardiopulmonary resuscitation. But a child can drown in minutes, even with an attentive caretaker nearby who knows that a swimming pool can be dangerous. Forty six per cent of young victims of pool drowning were missing for five minutes or less. ${ }^{5}$ Among children under 4 in the United States, drowning is the third most common cause of injury death. ${ }^{6}$ The death rate for drowning of 3.4 per 100000 in the United States is comparable with that of 3.5 per 100000 found in Australia. ${ }^{7}$ A fence around a pool or spa is an effective safeguard that helps prevent such tragedies. Homeowners who understand the risks of drowning may voluntarily erect a fence and keep it safely locked. Architects and designers may change their organizational practices and priorities by promoting four sided fencing. However, achieving lower drowning rates in a community is likely to require a broad based intervention such as the passage of a pool fencing ordinance. Such zoning changes are more likely to be implemented with a comprehensive approach. When the community is well educated, it is more inclined to support and adopt such changes. For example, a coalition of paramedics, relatives of drowning victims, real estate agents, firefighters, and child safety advocates can mobilize political support to ensure the passage of a fencing ordinance.

\section{Using the spectrum of prevention to} prevent childhood and adolescent injuries This section defines the six levels of the spectrum, beginning with a description of each method, followed by specific examples. An activity at any of the spectrum's six levels constitutes an intervention. However, when these initiatives are used in combination, the spectrum becomes a more transformative force for individual, community, and societal health. This inter-relatedness between levels of the spectrum, or "synergy", enables practitioners to maximize the result of any one prevention activity by strengthening the linkages between multiple efforts.

\section{(1) STRENGTHENING INDIVIDUAL KNOWLEDGE}

AND SKILLS

Strengthening individual knowledge and skills involves transferring information and knowhow to increase an individual's resources and capacity for preventing injury or disease. In a trusting relationship with a person who is perceived to have expertise or authority, even brief comments have a lasting impact, particularly when reinforced over time or through community norms and practices.

Physician advice, for example, has been associated with reductions in morbidity, mortality, risk behaviors, and risk factors and an increase in healthy behaviors. ${ }^{8}$ During checkups, physicians have the opportunity to ask children and their parents if they properly store medicines and household poisons and whether they use bicycle helmets. Such advice has been shown to increase the likelihood of bicycle helmet ownership for children. ${ }^{9}$ Responsibility for strengthening individual knowledge and skills need not be limited to physicians and human service professionals. Bicycle sales people can demonstrate how to properly fit a helmet for optimum safety. Many organizations and community agencies use individual skill building as the primary approach to help individuals learn healthy behaviors and change unhealthy habits.

One well known program designed to reduce alcohol related injuries is the "designated driver" program that employs high school students as peer counselors. Young people teach fellow students to understand the risks of drinking and driving. Peers help other students develop skills and commitment to designate drivers, refuse to ride with people who have been drinking, and abstain from drinking when they will be driving. This campaign aims to change individual behavior, and encourage healthy choices in an effort to reduce alcohol related traffic injuries. ${ }^{10}$ 
(2) PROMOTING COMMUNITY EDUCATION A community education approach aims to reach groups of people with information and resources for improving health. Community education is broadly targeted at groups or the population at large. Mass media campaigns have been shown to increase awareness, change attitudes, and provide a context in which other strategies can succeed, ${ }^{11}$ such as public policy change. ${ }^{12}$ Effective community education not only alerts individuals to new information, but also builds a critical mass of support for healthier behavior, norms, and policy change.

For example, "bike days", a community education approach in the field of unintentional injury engages police, retailers, local health practitioners. Bicycle enthusiasts conduct a variety of activities in schools and surrounding communities to promote cycling safety. "Bike days" events use performances by professional stunt bicyclists and "bike rodeos" to gain the enthusiasm of youngsters while teaching them the rules of the road and using the opportunity to distribute and check for correctly fitted helmets. Posters and the mass media promote the events, while educating and involving the public.

Increasingly worldwide, mass media is the primary vehicle for community education. When conducting events to increase community awareness about prevention, media coverage before, during, and after can reinforce the prevention message to a larger audience. In this way, media can build support for injury prevention issues by reflecting community involvement and activism. When communities employ media strategically, with the intention of creating change in institutions and policy, it is referred to as "media advocacy". This is an approach that helps people frame issues from the perspective that health and social problems will only be adequately addressed when all sectors of society - not just the individual-share responsibility for solutions. When communicated effectively to the media, public health solutions which emphasize shared responsibility gain the attention and support of legislators and policy makers, acting as a catalyst for policy change.

(3) EDUCATING PROVIDERS

Providers have influence within their fields of expertise and opportunities to transmit information, skills, and motivation to patients, clients, and colleagues. It is essential, therefore, that they receive education to improve their own understanding of prevention. Medical training has recently begun to place more emphasis on teaching doctors the value of advising children, adolescents, and parents about the injuries associated with unsafe practices. ${ }^{13}$ As a result, certain professionals (for example doctors, teachers, child care workers, etc) can be highly effective advocates for policy changes related to their job experiences.

By expanding the notion of provider, it is possible to mobilize a broader group in advancing prevention and promoting wellness. Since 1990, traffic reporters in northern and southern California have attended conferences where they learn that "injuries are no accident", and that they have a critical role in educating the public. As injury prevention specialists outline how most injuries are predictable, and therefore, preventable, traffic reporters learn that through "injury sensitive" reporting techniques they can transmit prevention messages to large numbers of people every day, and heighten public awareness of the problem.

In response to the needs of health care and educators, a training curriculum, Leadership Training in Violence Prevention, was developed to build the skills and capacities of providers. With funding from the United States Department of Health: Maternal, Child and Adolescent Health Division, and the Department of Education, the curriculum was a combination of pioneering courses taught at Harvard and University of California at Berkeley. In the first year, over 100 people representing all regions, and serving diverse populations of the United States were trained and agreed to conduct at least three trainings each in their communities. Evaluations analyzed by the Harvard School of Public Health (unpublished data) indicated that as a result they felt well prepared to perform their roles as trainers and more than three quarters have met or exceeded their training commitment. As a result, thousands more people have received training in violence prevention, nationally.

\section{(4) FOSTERING COALITIONS AND NETWORKS}

Fostering collaborative approaches brings together the participants necessary to assure an initiative's success. Coalitions and expanded partnerships are vital in successful public health movements including injury prevention. Coalitions increase the "critical mass" behind a community effort, help groups to trust one another, and reduce the likelihood of resource squandering through unnecessary competition among groups. ${ }^{14}$

Coalitions are useful for accomplishing a broad range of goals that reach beyond the capacity of any individual member organization. Like a jigsaw puzzle, each piece is important, and only when put together does the picture become clear. By working together, coalitions can conserve resources by reducing duplication and sharing expenses; foster cooperation between diverse sectors of society; and, increase the credibility and often the impact of their efforts.

The National Funding Collaborative, formed by foundations across the United States working to create a comprehensive violence prevention model, awarded violence prevention grants to 14 local collaboratives in cities and rural areas throughout the country. While each group works primarily in its own community, the collaboratives benefit from networking and mutual aid. Building Effective Coalitions: An Eight-Step Guide, expands upon this level of the spectrum and provides tips for building collaborations and partnerships. ${ }^{15}$

\section{(5) CHANGING ORGANIZATIONAL PRACTICES} Examining the practices of key organizations, such as law enforcement, health departments, 
and schools has potential for affecting the health and safety of the greater community. This is usually the least understood and most frequently ignored component of the spectrum. Yet this level has enormous potential. By changing its own internal regulations and norms, an organization can affect the health and safety of its members.

A school that has had several serious child pedestrian injuries may recognize that school practices have a potential role in preventing some of these injuries. Hiring crossing guards represents an opportunity to change the practice of the school and serve to increase child safety. Altering organizational practices can positively effect the work environment, while benefiting staff and the people they serve.

Often changes in organizational practice are initiated as a result of change in legislation. For instance, after the passage of United States regulations that set the minimum drinking age at 21 , new law enforcement procedures, such as sobriety checkpoints, have been attributed with saving an estimated 15667 lives between 1975 and $1995 .{ }^{16}$

(6) INFLUENCING POLICY AND LEGISLATION Changes in local, state, and national laws, as well as the adoption of formal policies by boards and commissions, fall under the umbrella of policy and legislation. Influencing policy usually presents the opportunity for the broadest improvement in health outcomes. Both institutional and legal policies can affect large numbers of children and adolescents. In some cases, laws and policies already exist that could protect public health and safety, but an additional law, change in policy, better enforcement, or change in an organization's practices may be necessary to ensure its effectiveness.

For instance, a parents' group concerned about playground safety might approach the local government with their concerns about potentially unsafe surfaces that cause injuries when children fall. After an assessment of playground surfaces in local parks, the group proposes legislation to upgrade to safer surfaces and implement regulations on playground equipment. Through their assessment, they can lay the groundwork for building support and influencing policy that will improve playground safety.

Requiring safe practices, implementing safety standards, and encouraging the use of safety equipment can prevent unintentional injuries. Recent data demonstrate that California's mandatory helmet law for bikes and motorcycles has dramatically reduced the number of serious and fatal head injuries. ${ }^{17}$

Several United States cities and counties have recently passed laws that regulate the conditions under which guns may be bought and sold. New laws that reduce the availability of deadly weapons to children are helping to lower the toll of firearm death and injury. Equally important, these victories are building momentum for a more comprehensive set of state and national gun regulations aimed at preventing firearm injuries.

\section{Data and evaluation}

Data and evaluation inform all levels of the spectrum. Any proposed activity should be based on data showing (1) the issue is important, (2) the target population is appropriate, and (3) the intervention is promising. To develop a successful approach it is essential to first review the data and determine an appropriate set of objectives. During implementation, ongoing evaluation of the overall approach and the individual activities at each level of the spectrum will provide information necessary for making ongoing adjustments to the activities that are best suited to meet overall objectives.

For example, after a train hit a young boy, a community was prepared to mobilize to prevent such occurrences. However, after reviewing child injury data from that community, it became clear that train related injuries were extremely rare, and many more children were killed by cars. As a result, a coalition formed to promote the safety of child pedestrians. While the coalition initially focused efforts on young children going to and from school, it later realized that children were at greater risk later in the day around neighborhood parks, and therefore expanded its efforts to include neighborhood outreach. In this way, data and evaluation can be used to inform and modify prevention efforts.

At the design stage it is helpful to identify ways to measure success and gather input from participants and the community. Successful prevention also requires assessment that takes into account the causative factors of childhood and adolescent injuries and the resiliency factors that protect youth from being injured. Determining shifts in community norms, improved networks, the effectiveness of coalitions, and enhanced community awareness are difficult to measure, but increasingly, process evaluations and survey tools are being developed to measure these changes. ${ }^{18}$

While there are anecdotal endorsements of the spectrum in a number of fields, including injury prevention, it should be noted that the tool itself has not been evaluated; its value comes from the way it has helped practitioners, individually and in groups, to structure multifactored initiatives. In this way, it resembles other tools designed to aid practitioners in conceptualizing and shaping their work. Its evaluation would be an important step toward furthering its credibility and utility.

\section{Conclusion}

The spectrum of prevention is a framework that delineates a systems approach to prevention practice. The spectrum has been applied to health problems in communities worldwide. In particular, the spectrum is being used increasingly in injury prevention practice. For example, the National Highway Traffic Safety Administration and the World Health Organization have both made use of this tool during professional trainings in comprehensive approaches and strategy development. State funded projects in California in injury 
prevention, nutrition, and physical activity have been required to use the spectrum of prevention in program design and evaluation. The spectrum has been used in "requests for proposals" as a way to evaluate the merit of competing applicants.

While there has been a great deal of positive movement in injury prevention, results have not been maximized through sustained, strategic efforts. Often practitioners work in areas that are most familiar to them, such as community education and individual skill building. The spectrum of prevention is a tool that enables practitioners to move beyond a primarily educational approach to achieve broad impact through multifaceted activities. It can aid practitioners and policy makers in thinking through, evolving, and strategically developing prevention programming efforts.

As communities seek to address increasingly complex social and health issues they will face the challenge of devising new services and programs in response to injuries until they are committed to promoting prevention. While every injury or death cannot be prevented, when systematic methodology, like the spectrum of prevention, is applied and an overall strategy developed, prevention efforts have an excellent chance for success. Childhood and adolescent injuries and deaths, once thought of as accidents, can be prevented. A good strategy solves multiple problems, saves lives and money, reduces suffering, and enhances the prospects for community well being.

The authors would like to thank Manal Aboelata for editing the article.
1 Rivara FP, Mueller BA. The epidemiology and causes of childhood injuries. Fournal of Social Issues 1987;43:13-31.

2 Swift MS. Alternative teaching strategies, helping behaviorally troubled children achieve: a guide for teachers and psychologists. Illinois: Research Press, 1975.

3 Haddon W. Escape of tigers: an ecologic note. Am $\mathcal{F}$ Public Health 1970;60:2229-34.

4 Albee GW. Psychopathology, prevention, and the just society. Fournal of Primary Prevention 1983;4:5-40.

5 US Consumer Product Safety Commission. Child drowning study: a report on the epidemiology of drowning in residential pools to children under five. Washington, DC: Government Printers Office, 1987.

6 Ellis AE, Trent RB. Swimming pool drownings and near-drownings among California preschoolers. Public Health Rep 1997;112:73-7.

7 Baker SP. International comparisons: useful or odious? Inj Prev 1997;3:3.

8 Gruninger UJ. Patient education: an example of one-to-one communication. F Hum Hypertens 1995;9:15-25.

9 Schneider ML, Ituarte P, Stokols D. Evaluation of a community bicycle helmet promotion campaign: what works and why. American fournal of Health Promotion 1993; 7:281-7.

10 Roper Starch Worldwide Inc. Roper reports. Storrs, CT: Roper Center for Public Opinion, 1998 (March). Report No 98-3.

11 Rootman I. Preventing alcohol problems: a challenge for health promotion. Health Education 1985;24:2-7.

12 Jernigan DH, Wright PA. Media advocacy: lessons from community experiences. F Public Health Policy 1996;17: 306-30.

13 Richmond R, Kehoe L, Heather N, et al. General practitioners' promotion of healthy life styles: what patients think. Australian and New Zealand fournal of Public Health 1996;20:195-200.

14 National Committee for Injury Prevention and Control. Injury prevention: meeting the challenge. Am $\mathcal{F}$ Prev Med 1989; 5:98.

15 Cohen L, Baer N, Satterwhite P. Developing effective coalitions: an eight-step guide. Injury Awareness \& Prevention Center News 1991;4:10.

16 Bonnie RJ, Fulco CE, Liverman CT, eds. Reducing the burden of injury: advancing prevention and treatment. Washington, DC: National Academy Press, 1999.

17 Krause JF, Peek C, McArthur DL, et al. The effect of the 1992 California motorcycle helmet use law on motorcycle 1992 California motorcycle helmet use law on motorcy
crash fatalities and injuries. $7 A M A$ 1994;272:1506-11.

18 Fawcett, SB, Lewis, RK, Paine-Andrews, A, et al. Evaluating community coalitions for the prevention of substance abuse: the case of Project Freedom. Health Education \& Behavior 1997;24:812-28.

\section{Editorial Board Member: brief biography}

\section{JESS F KRAUS}

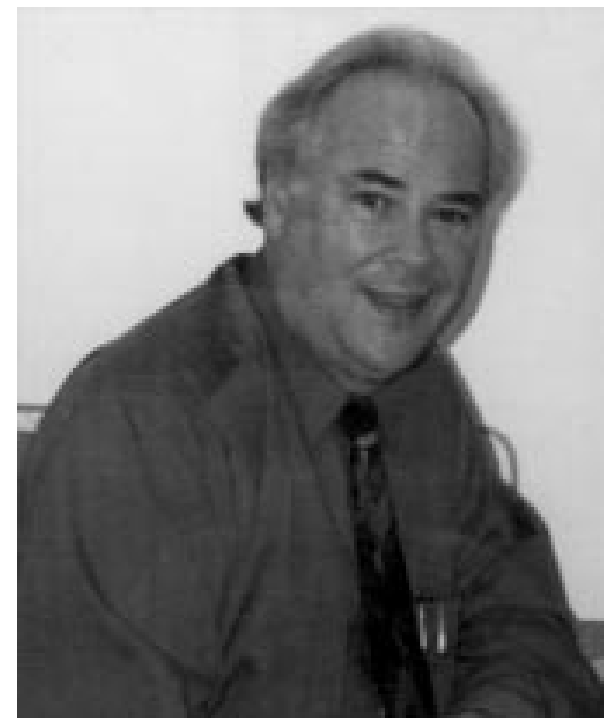

Jess $\mathrm{F}$ Kraus, $\mathrm{MPH}, \mathrm{PhD}$, has been engaged in injury research since the mid-1960s. He is currently Professor of Epidemiology and Director of the Southern California Injury Prevention Research Center at the University of California Los Angeles.

$\mathrm{He}$ has held numerous appointed and elected positions in many professional societies and organizations such as the US Department of Transportation Motor Vehicle Safety Research Advisory Committee and the World Health Organization Expert Advisory Panel on Accident Prevention.

He has undertaken studies on motorcycle crashes, pedestrian injuries, work related trauma, and brain and spinal cord injuries. He has more than 175 publications in the field and teaches five graduate courses on injury epidemiology. 